\title{
Effect of Biomechanical and Anatomic Characteristics on Patient-Reported Outcomes Following Total Hip Arthroplasty
}

\author{
Lauren Pitz BS, ${ }^{1}$ Braeden W. Estes BS, ${ }^{1}$ \\ Evan R. Deckard BSE, ${ }^{2}$ R. Michael Meneghini MD ${ }^{2}$ \\ ${ }^{1}$ Indiana University School of Medicine \\ ${ }^{2}$ Indiana University School of Medicine, Department of Orthopaedic Surgery \\ Background and Hypothesis: The success of total hip arthroplasty (THA) is \\ often reported in terms of infection and dislocation rates. While studies have \\ examined the effect of acetabular cup position, anteroposterior (AP) femoral \\ stem alignment, changes in leg length and femoral stem offset on dislocation \\ rates, few studies have investigated the effect of these biomechanical \\ parameters on patient-reported outcome measures (PROMS). The purpose of \\ this study was to evaluate how PROMS may differ by THA component placement \\ in a consecutive series of primary THAs. Awareness of the importance of implant \\ positioning may lead to improved surgical technique and optimized PROMS.
}

Experimental Design or Project Methods: 933 consecutive posterolateral approach primary THAs performed between 2011 and 2018 by one surgeon were retrospectively reviewed. Acetabular cup abduction, femoral stem alignment, changes in leg length and total femoral offset were measured on APview radiographs. Prospectively collected Hip Disability and Osteoarthritis Outcome Score/HOOS Jr., University of California Los Angeles/UCLA Activity Level, and satisfaction (5-point Likert scale) were evaluated at minimum oneyear.

Results: 743 THAs were analyzed. Mean age and BMI were 64 years and 31 $\mathrm{kg} / \mathrm{m}^{2}$, respectively. After multivariate analysis, females with neutral to valgus stem placement $(p=0.020)$ and patients with neutral to valgus stem placement regardless of lumbar pain $(p=0.034)$ were more satisfied. In addition, patients with lumbar pain $(p<0.001)$ and patients with high BMl in combination with increased change in femoral offset $(p=0.056)$ had lower overall HOOS Jr. scores. Interestingly, change in leg length was not a significant predictor of any PROMS (power $[1-\beta] \geq 88.4 \%$ ).

Conclusion and Potential Impact: AP stem alignment may play a role in increased activity level and satisfaction. In addition, high BMI in combination with increased change in femoral offset negatively influenced HOOS Jr. scores. Unsurprisingly, the presence of lumbar pain continues to negatively affect PROMS. Further research is warranted on the influence of THA component placement, spinopelvic parameters, and PROMS. 